\title{
Influência da armadura transversal recomendada pelas normas NBR 6118 (2007), ACI 318 (2005) e Eurocode 2-1 (2003) no comportamento de vigas de concreto armado
}

\author{
Agleílson Reis Borges ${ }^{(1)}$, Dênio Ramam Carvalho de Oliveira $^{(2)}$
}

\begin{abstract}
Resumo
Para avaliar a influência da taxa mínima de armadura transversal no comportamento de vigas de concreto armado com seção retangular foram analisadas experimentalmente, no Laboratório de Engenharia Civil da Universidade Federal do Pará (UFPa ), doze vigas com dimensões de $2.200 \mathrm{~mm}$ para o comprimento (l), $250 \mathrm{~mm}$ para a altura $(\mathrm{h})$ e bases $\left(\mathrm{b}_{\mathrm{w}}\right)$ variando de $120 \mathrm{a} 420 \mathrm{~mm}$, com incremento nesta dimensão igual a 60 $\mathrm{mm}$. Todas as vigas apresentaram taxas geométricas de armadura de flexão iguais e foram submetidas ao ensaio de Stuttgard, com vão livre de $2.000 \mathrm{~mm}$. São apresentados e analisados os resultados experimentais obtidos para as cargas últimas, modos de ruptura, deformações da armadura de flexão, deformações no concreto e deslocamentos verticais. Os resultados experimentais foram comparados com as estimativas das normas NBR 6118 (2007) e divergências significativas foram observadas.
\end{abstract}

Palavras-chave: Concreto armado. Viga. Cisalhamento.

1 Doutorando, Escola Politécnica da Universidade de São Paulo, departamento de estruturas e geotécnica, Av. Prof. Almeida Prado, 83, tv. 2, Cidade Universitária-São Paulo-São Paulo, Brasil - CEP: 05508-900. E-mail: agleilson@ usp.br(enviar correspondência para este autor)

2 Professor Doutor, Faculdade de Engenharia Civil, Universidade Federal do Pará. Campus Universitário do Guamá, Rua Augusto Corrêa, Número 01, Guamá-Belém-Pará-Brasil- CEP: 66075-970. E-mail: denio@ufpa.br.

http://dx.doi.org/10.5335/rsee.v10i1.2379

24 


\section{Introdução}

No dimensionamento de elementos estruturais solicitados por esforços cisalhantes é imprescindível uma analise minuciosa a respeito da ductilidade da mesma, para que em caso de iminente sinistro tenha-se uma estrutura que "avise" quando estiver próxima de sua resistência última, tanto para dar o devido tempo para a evacuação do local caso necessário, quanto para se tomar as medidas necessárias para que a estrutura seja reforçada.

Segundo Garcia (2002), as armaduras transversais mínimas de vigas devem ser providas visando fazer com que, na eventualidade da existência de sobrecargas não previstas, as vigas não apresentem ruptura brusca, assim que ocorra a fissuração diagonal, limitar a inclinação das bielas e a abertura de fissuras inclinadas e evitar a flambagem da armadura longitudinal comprimida.

As normas para cálculo de estruturas de concreto armado estabelecem armaduras transversais mínimas, mas nem todas esclarecem seus objetivos e nenhuma cita em que se baseou a definição dessa armadura. Na norma ACI-318 (2005) consta que a armadura transversal mínima restringe o desenvolvimento da fissuração inclinada, aumenta a ductilidade da viga e provê aviso de possível ruptura, sendo de particular importância se o membro é submetido a sobrecargas não previstas.

Tendo como motivação a avaliação da taxa de armadura transversal mínima, este artigo versa sobre a análise experimental de vigas com taxa de armadura mínima utilizando-se de vigas com diferentes $b_{w}$ e expõe, além de analisar, uma nova formulação para o cálculo da resistência última ao esforço cortante de vigas de concreto armado com armadura transversal mínima.

\section{Estimativas normativas}

A Tabela 1 apresenta as formulações adotadas por cada uma das normas analisadas para o dimensionamento ao esforço cortante em vigas de concreto armado com estribos perpendiculares ao eixo longitudinal da peça. A NBR 6118 (2007) e o EUROCODE 2-1 (2003) utilizam como princípio, a treliça generalizada de Mörsch. Estas normas permitem que o ângulo de inclinação $\alpha$ das bielas de tração (armadura transversal) varie de $45^{\circ}$ a $90^{\circ}$ e prescrevem $45^{\circ}$ como o valor máximo para o ângulo $\theta$ da biela comprimida de concreto. O EUROCODE 2-1(2003) permite a utilização de $\theta$ com um valor mínimo de $21,8^{\circ}$ enquanto a NBR 6118 (2007) limita este valor mínimo em $30^{\circ}$. O valor do ângulo $\theta$, é arbitrado pelo projetista. Na verificação da ruptura por tração diagonal, observa-se que o EUROCODE 2-1(2003) não leva em consideração a contribuição dos mecanismos complementares à treliça, isto é, a armadura transversal absorve integralmente todo o esforço cortante de cálculo. O ACI 318(2005) por sua vez baseia-se na analogia clássica da treliça, com o ângulo de inclinação da biela comprimida $\theta$ fixo a $45^{\circ}$. 
Tabela 1: Critérios de Dimensionamento ao Esforço Cortante em Vigas com $\alpha=90^{\circ}$

\begin{tabular}{|c|c|c|}
\hline Norma & $\begin{array}{l}\text { Verificação da Tensão na } \\
\text { Biela de Concreto }\end{array}$ & $\begin{array}{l}\text { Verificação da Tração Diagonal } \\
\tau_{\text {Calc }} \leq \tau_{c}+\tau_{s w}\end{array}$ \\
\hline $\begin{array}{c}\text { NBR } 6118(2007) \\
\text { Modelo I } \\
\text { fck } \leq 50 \mathrm{MPa}\end{array}$ & $\begin{array}{c}\tau_{s d} \leq \tau_{R d 2} \\
\tau_{R d 2}=0,27 \alpha_{v 2} f_{c d} \\
\alpha_{v 2}=\left(1-\frac{f_{c k}}{250}\right)\end{array}$ & $\begin{array}{c}\tau_{s d} \leq \tau_{R d 3}=\tau_{c}+\tau_{s w} \\
\tau_{c}=\tau_{c 0}=0,126\left(f_{c k}^{2 / 3}\right) \\
\tau_{s w}=0,90 \rho_{w} f_{y w d}\end{array}$ \\
\hline $\begin{array}{c}\text { NBR } 6118(2007) \\
\text { Modelo II } \\
\text { fck } \leq 50 \mathrm{MPa} \\
30^{\circ} \leq \theta \leq 45^{\circ}\end{array}$ & $\begin{array}{c}\tau_{s d} \leq \tau_{R d 2} \\
\tau_{R d 2}=0,27 \alpha_{v 2} f_{c d} \operatorname{sen} 2 \theta \\
\alpha_{v 2}=\left(1-\frac{f_{c k}}{250}\right)\end{array}$ & $\begin{array}{c}\tau_{s d} \leq \tau_{R d 3}=\tau_{c}+\tau_{s w} \\
\tau_{c}=\tau_{c 1}=\tau_{c 0}\left(\frac{\tau_{R d 2}-\tau_{s d}}{\tau_{R d 2}-\tau_{c 0}}\right) \\
\tau_{s w}=0,90 \rho_{w} f_{y w d} \operatorname{cotg} \theta\end{array}$ \\
\hline $\begin{array}{c}\text { EUROCODE } 2-1(2003) \\
\text { fck }>60 \mathrm{MPa} \\
21,8^{\circ} \leq \theta \leq 45^{\circ}\end{array}$ & $\begin{array}{c}\tau_{R d} \leq \tau_{R d, m a ́ x} \\
\tau_{R d, m a ́ x}=0,45 v_{1} f_{c d} \operatorname{sen} 2 \theta \\
f_{c k} \leq 60 M P a: v_{1}=0,60 \\
v_{1}=\left(0,90-\frac{f_{c k}}{200}\right)\end{array}$ & $\begin{array}{c}\tau_{R d} \leq \tau_{R d, s y} \\
\tau_{c}=0 \\
\tau_{s w}=0,90 \rho_{w} f_{y w d} \operatorname{cotg} \theta\end{array}$ \\
\hline $\begin{array}{c}\mathrm{ACl} 318(2005) \\
\mathrm{fck}<69 \mathrm{MPa} \\
\theta=45^{\circ}\end{array}$ & $\begin{array}{c}\tau_{u} \leq 0,75 \tau_{n} \\
\tau_{u} \leq 0,83 \sqrt{f_{c}}\end{array}$ & $\begin{array}{c}\tau_{n}=\tau_{c}+\tau_{y} \\
\tau_{c}=0,17 \sqrt{f_{c}} \\
\tau_{s}=\rho_{w} f_{y w k} \leq 0,66 \sqrt{f_{c}}\end{array}$ \\
\hline
\end{tabular}

Onde:

$\mathrm{A}_{\mathrm{sw}}=$ armadura transversal;

$\rho_{\mathrm{w}}=$ é a taxa de armadura transversal, igual a Asw(bw/s);

$\mathrm{f}_{\mathrm{ck}}=$ é a resistência do concreto em $\mathrm{Mpa}\left(\boldsymbol{f}_{c k}>60 \mathrm{MPa}\right.$ : $)$;

$\theta$ é o ângulo de inclinação das bielas comprimidas;

$\mathrm{s}=$ é o espaçamento dos estribos, em $\mathrm{cm}$;

bw = é a menor largura da seção, medida em cm;

fywd = é a tensão de escoamento de cálculo na armadura transversal em $\mathrm{MPa}$;

$\tau_{\mathrm{R}}=$ é a tensão resistente;

$\tau_{\mathrm{S}}=$ é a tensão solicitante;

$\alpha=$ é o ângulo de inclinação da armadura transversal em relação ao eixo longitudinal da viga;

$\alpha v 2$ = é um coeficiente adimensional, que representa o índice de fragilidade do concreto, dado por (1-fck/250), sendo fck em MPa. 


\subsection{Armadura Miníma}

Garcia (2002) define taxa de armadura transversal mínima como aquela necessária para que a viga não tenha ruptura frágil após a força cortante ter alcançado o valor do cortante de fissuração diagonal. As fórmulas de taxas de armadura transversal mínima propostas nos códigos atuais são basicamente empíricas, em geral não incluem todos os parâmetros relevantes, levando a valores que diferem muito entre sim. Para este trabalho foi utilizada a formulação da NBR 6118 (2007), mostrada na Equação 1.

$$
\rho_{s w, \min }=\frac{A_{s w}}{b_{w} \cdot s \cdot \cos \theta} \geq \frac{0,06 \cdot f_{c k}^{2 / 3}}{f_{y s w} \cdot \operatorname{sen} \alpha}
$$

Onde:

Asw $=$ armadura transversal;

$\rho \mathrm{w}, \mathrm{min}=$ é a taxa de armadura transversal mínima;

fck = é a resistência do concreto em $\mathrm{MPa}$;

$\theta=$ é o ângulo de inclinação das bielas comprimidas;

$\mathrm{s}=$ é o espaçamento dos estribos, em $\mathrm{cm}$;

bw = é a menor largura da seção, medida em cm;

fyw = é a tensão de escoamento de cálculo na armadura transversal em MPa;

$\alpha=$ é o ângulo de inclinação da armadura transversal em relação ao eixo longitudinal da viga.

\section{Programa Experimental}

\subsection{Características das Vigas}

Para avaliar a influência da taxa mínima de armadura transversal (Asw) para diferentes valores de $b_{w}$ no comportamento último de vigas de concreto armado com seção retangular, analisaram-se experimentalmente 6 vigas de concreto armado com dimensões de $2200 \mathrm{~mm}$ para o comprimento (L), $250 \mathrm{~mm}$ para a altura (h) e bases (bw) que variaram de 120 a $420 \mathrm{~mm}$ com valor de incremento constante e igual a $60 \mathrm{~mm}$ Estas e outras especificações das vigas são mostrados nas Figuras 1 e 2, e outras especificações relevantes das peças ensaiadas são apresentados na Tabela 2. 


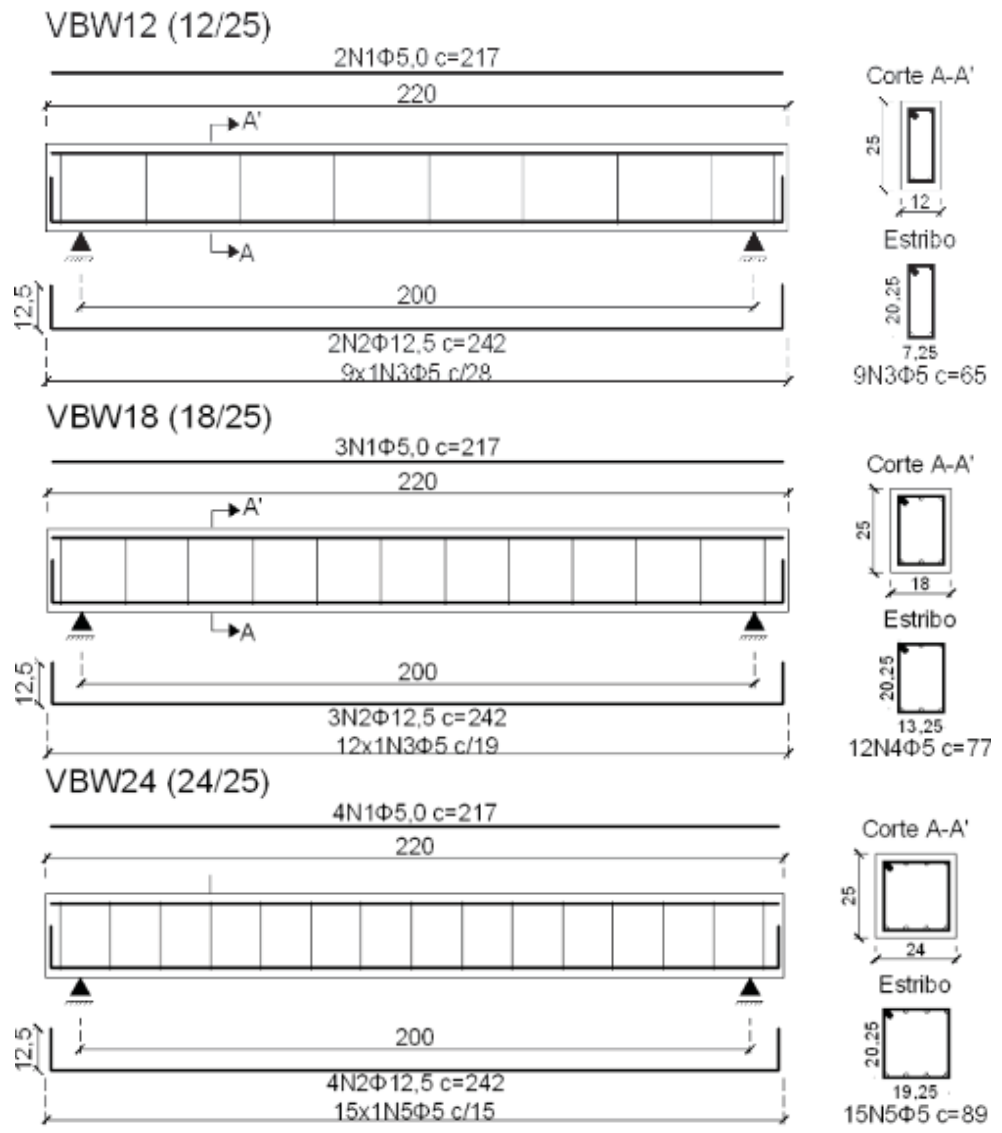

Figura 1: Armadura das vigas VBW12, VBW18 e VBW24.
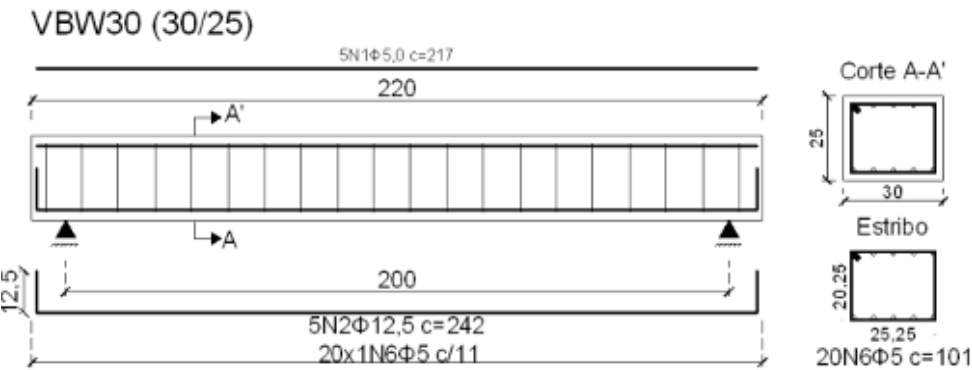


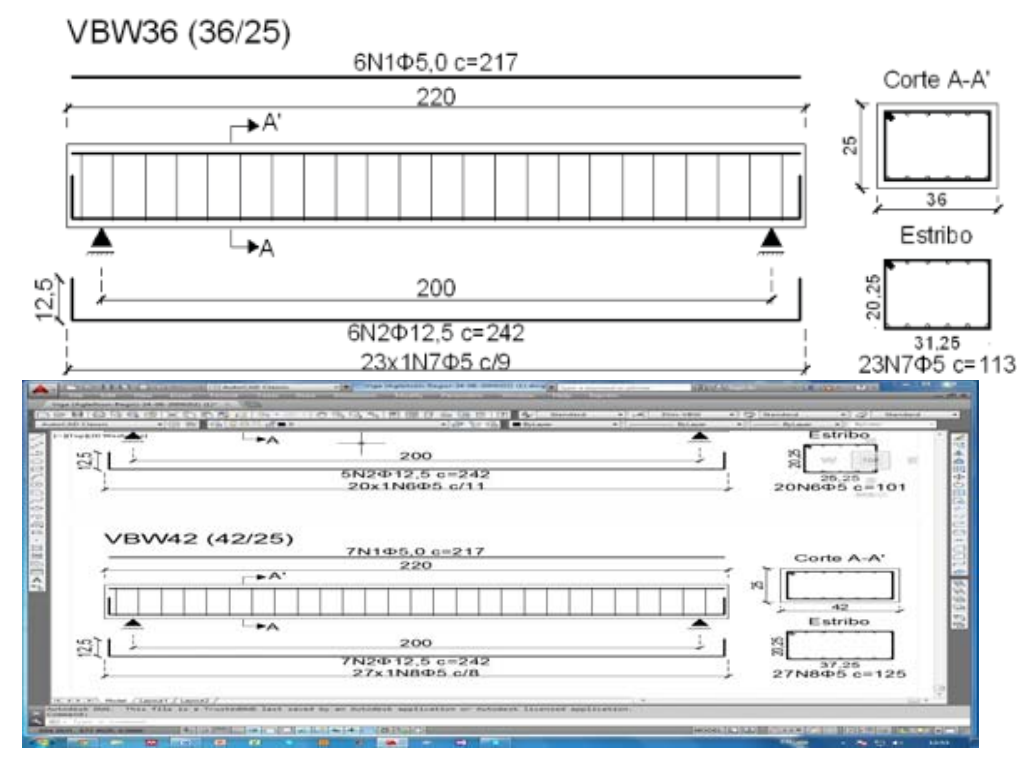

Figura 2: Armadura das vigas VBW30, VBW36 e VBW42.

Tabela 2: Características geométricas das peças.

\begin{tabular}{c|c|c|c|c}
\hline Viga & $\mathrm{L}(\mathrm{mm})$ & $\mathrm{bw}(\mathrm{mm})$ & $\mathrm{h}(\mathrm{mm})$ & $\mathrm{d}(\mathrm{mm})$ \\
\hline VBW12 & & 120 & & \\
VBW18 & & 180 & & \\
VBW24 & 2200 & 240 & 250 & 230 \\
VBW30 & & 300 & & \\
VBW36 & & 360 & & \\
VBW42 & & 420 & & \\
\hline
\end{tabular}

\subsection{Instrumentação}

Para medir a flecha no centro do vão da viga durante os ensaios foi utilizado um deflectômetro analógico com precisão de $0,01 \mathrm{~mm}$. Já as deformações do concreto e das armaduras de flexão e cisalhamento foram monitoradas com extensômetros elétricos de resistência (EER). No concreto foram utilizados extensômetros elétricos de resistência (EER-comp), com $8 \mathrm{~mm} \times 95 \mathrm{~mm}$, fixados no centro da face superior da viga, para medir as deformações máximas de compressão na peça. $\mathrm{Na}$ armadura de flexão foram utilizados extensômetros, EER-flex, medindo 2,8 $\mathrm{mm}$ x 9,4 $\mathrm{mm}$ posicionados no centro de pelo menos uma de suas barras, os extensômetros utilizados para monitorar os estribos, EER-cis I, EER-cis II e EER-cis III, possuíam dimensões idênticas as dos extensômetros utilizados para o monitoramento a flexão, a Figura 3 mostra o esquema do posicionamento dos extensômetros nas vigas. Foi utilizado um sistema digital para 
aquisição de dados para a leitura dos extensômetros elétricos, composto por um módulo do equipamento de aquisição de dados Almemo e um laptop. A Figura 4 mostra os equipamentos utilizados na monitoração das deformações e flechas das vigas.
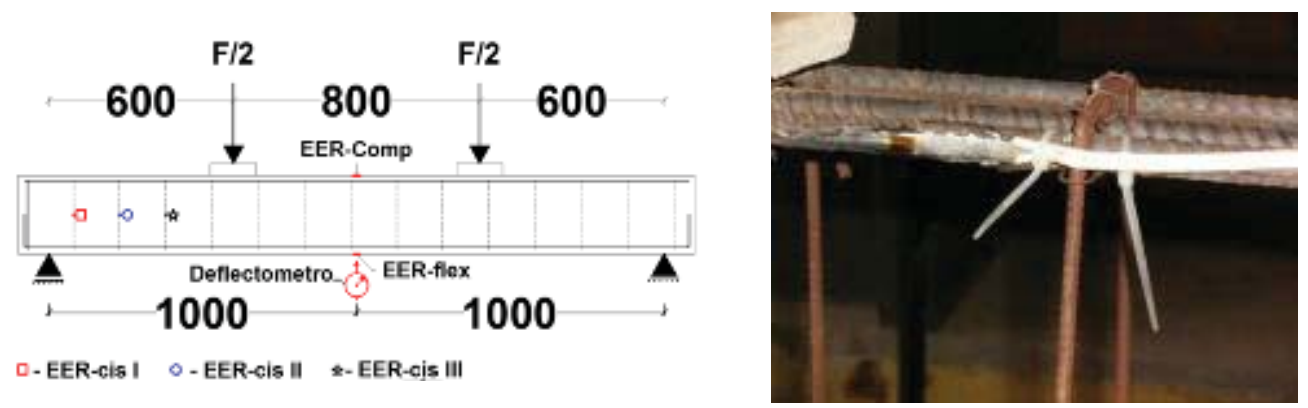

Figura 3: Instrumentação das vigas.
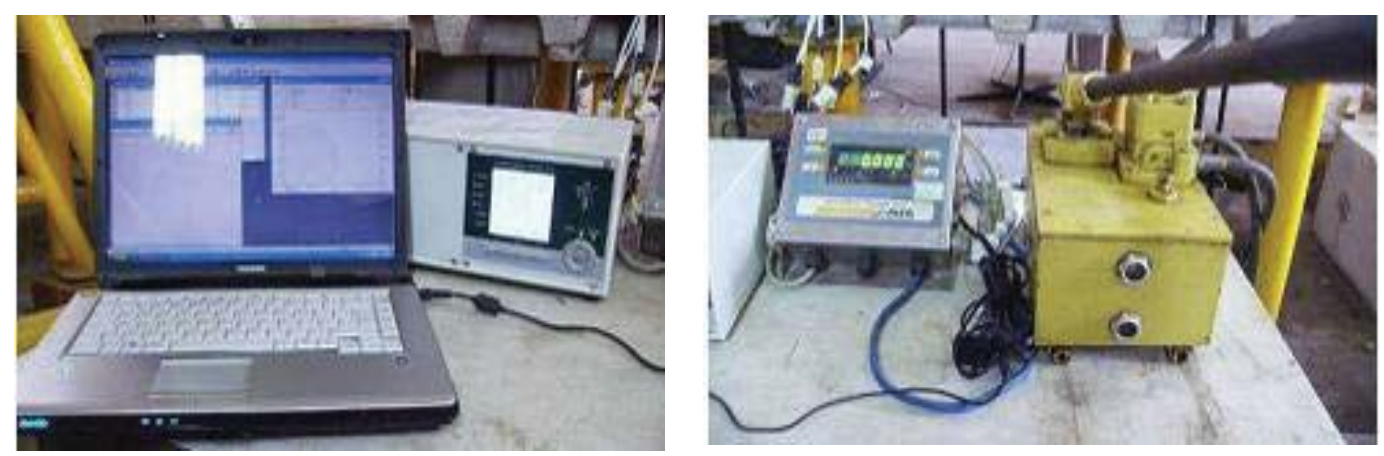

Figura 4: Equipamentos utilizados nos ensaios.

\subsection{Sistema de Ensaio}

O sistema de Ensaio do Laboratório de Engenharia Civil (LEC) da Universidade Federal do Pará possui um sistema de reação rígido, composto por um pórtico metálico de reação; um cilindro hidráulico para aplicação do carregamento; uma bomba hidráulica para acionar o cilindro; uma célula de força com leitora e precisão de $1 \mathrm{kN}$. Também foi utilizada uma viga metálica de perfil I para distribuir a força aplicada pelo cilindro hidráulico em dois pontos eqüidistantes dos apoios. Para o apoio das peças foram usados blocos de concreto e apoios específicos para garantir o equilíbrio isostático das peças.

O carregamento foi aplicado manualmente por meio de uma bomba hidráulica em etapas de $5 \mathrm{kN}$, no sentido de cima para baixo. Entre cada etapa do carregamento foi 
realizado o mapeamento das fissuras e a aferição das deformações do aço e do concreto. $\mathrm{O}$ sistema de ensaio consistiu basicamente na montagem de um sistema de Stuttgart para ensaios à flexão. A distância central entre as forças foi de $0,8 \mathrm{~m}$ e o vão ensaiado foi de 2,0 m, conforme mostram as Figuras 5 e 6 .

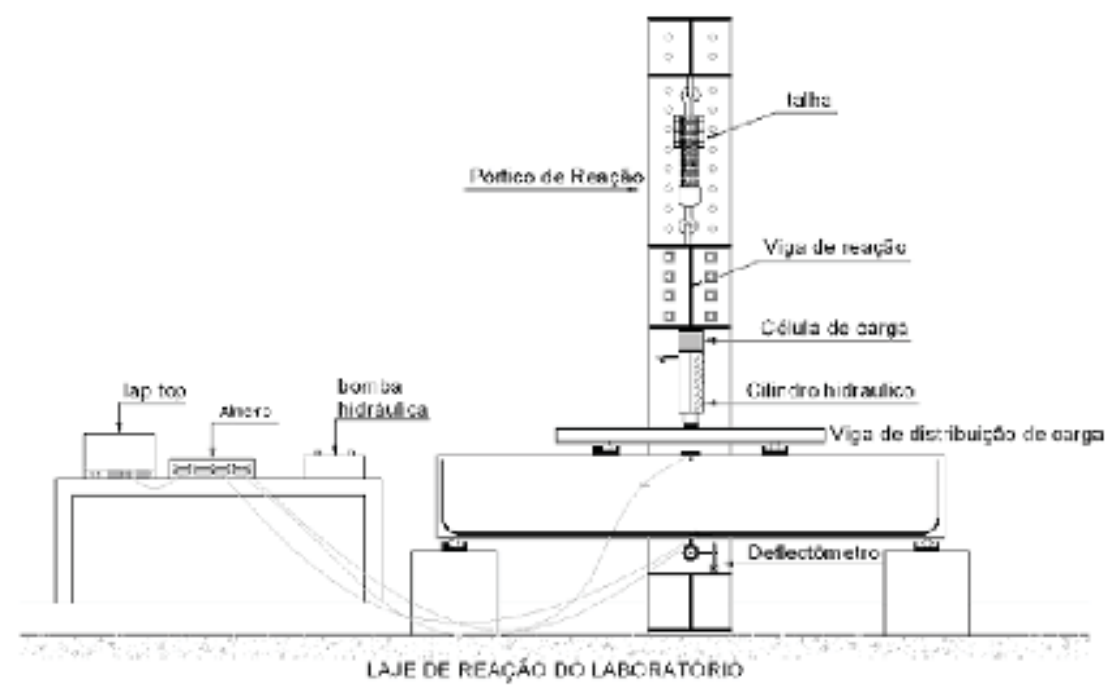

Figura 5: Croquis do sistema de ensaio.

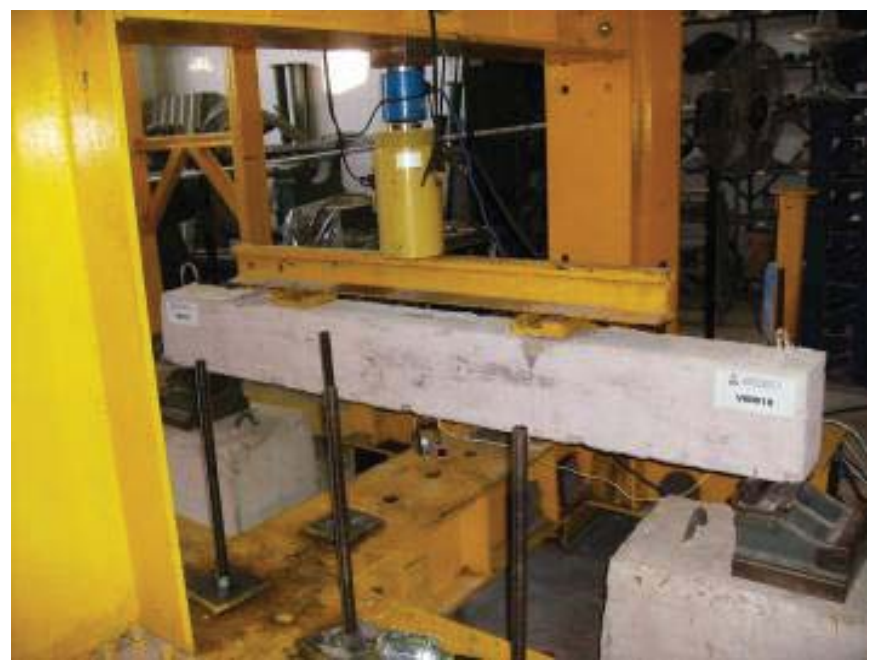

Figura 6: Sistema de ensaio e aquisição de dados. 


\section{Resultados}

\subsection{Propriedades dos Materiais}

\subsubsection{Concreto}

O concreto utilizado nas vigas foi dosado em central com cimento CPII-Z-32 e foram colhidos 6 corpos-de-prova (CP) cilíndricos de $150 \mathrm{~mm}$ x $300 \mathrm{~mm}$. Em 3 CPs foram realizados ensaios de compressão axial, segundo os parâmetros da NBR 5739 (2007), para determinação da resistência do concreto à compressão e outros 3 corpos-de-prova foram utilizados para determinação do módulo de elasticidade, obtido de acordo com a NBR 8522 (2008). Os resultados obtidos experimentalmente aos 28 dias para as resistências são apresentados na Tabela 3.

Tabela 3: Propriedades mecânicas do concreto.

\begin{tabular}{c|c|c}
\hline Corpo-de-prova & fc (MPa) & $\mathrm{E}_{\text {sec }}(\mathrm{GPa})$ \\
\hline 01 & 22,3 & 32,1 \\
02 & 23,4 & 30,5 \\
03 & 20,2 & 30,9 \\
Média & 22,0 & 31,2 \\
\hline
\end{tabular}

\subsubsection{Aço}

As barras de aço de diâmetros $12,5 \mathrm{~mm}$ e de $5,0 \mathrm{~mm}$ utilizadas nas armaduras de flexão e cisalhamento das vigas, respectivamente, pertenciam ao mesmo lote. Foram retiradas três amostras de cada bitola para realização dos ensaios de tração, seguindo as orientações da NBR ISO 6892 (2002), com a finalidade de se obter as propriedades mecânicas dos aços. As curvas tensão x deformação específica são mostradas na Figura 7 e os resultados são apresentados na Tabela 4.
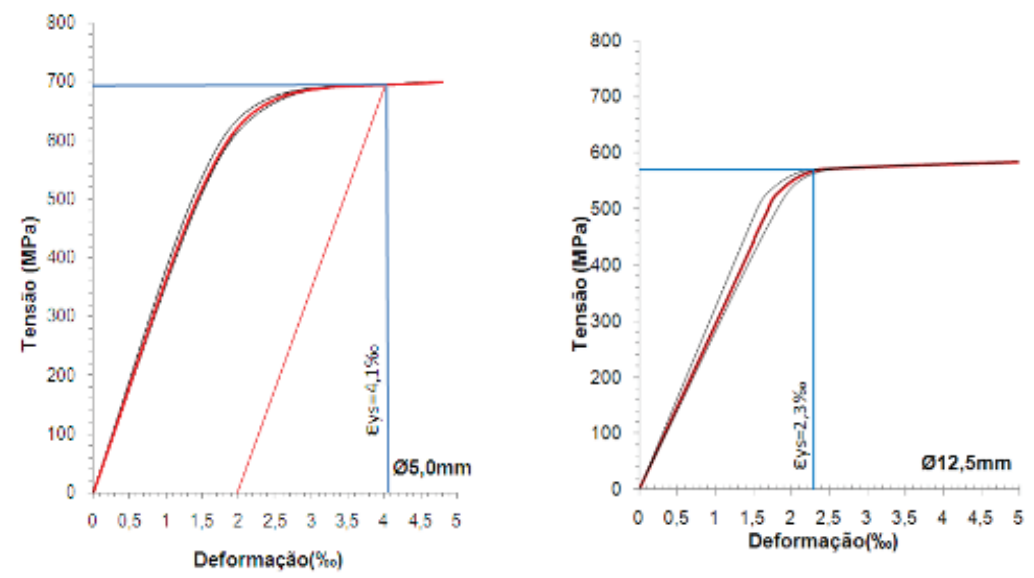

Figura 7: Diagrama tensão-deformação para as barras de aço. 
Tabela 4: Propriedades das barras de aço.

\begin{tabular}{c|c|c|c|c|c}
\hline$\varnothing(\mathrm{mm})$ & Área $(\mathrm{mm} 2)$ & fy $(\mathrm{kN})$ & fys $(\mathrm{MPa})$ & $\varepsilon_{\mathrm{ys}}(\% \circ)$ & fu (MPa) \\
\hline 5,0 & 20,0 & 13,6 & 695,0 & 4,1 & 709,2 \\
12,5 & 122,6 & 71,7 & 585,0 & 2,3 & 605,0 \\
\hline
\end{tabular}

\subsection{Deslocamentos Verticais}

Os deslocamentos monitorados foram analisando a partir das curvas Força Aplicada x Deslocamento, para cada viga, geradas com as leituras realizadas durante os ensaios. Na Figura 8, é possível perceber que as vigas ganham rigidez com o aumento da base das vigas $\left(b_{w}\right)$ o que era esperado, confirmando assim a teoria utilizada para $o$ seu dimensionamento.

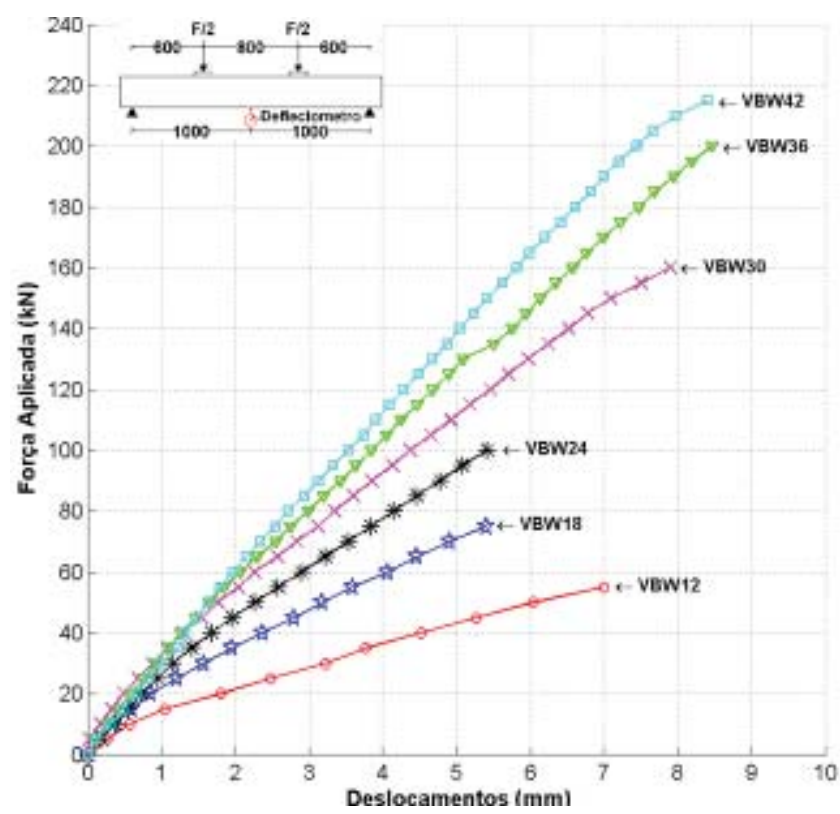

Figura 8: Deslocamentos verticais das vigas.

\subsection{Deformação Específica do Concreto}

Com o aumento gradativo do comprimento da base da viga (bw) das vigas pode-se observar um alivio na intensidade da solicitação na zona comprimida do concreto, para a região monitorada, podendo-se observar também o aumento da rigidez da viga com esse acréscimo no valor do bw, diminuindo assim a inclinação da curva Força Aplicada x Deformação, as curvas das vigas ensaiadas são mostradas na Figura 9. 


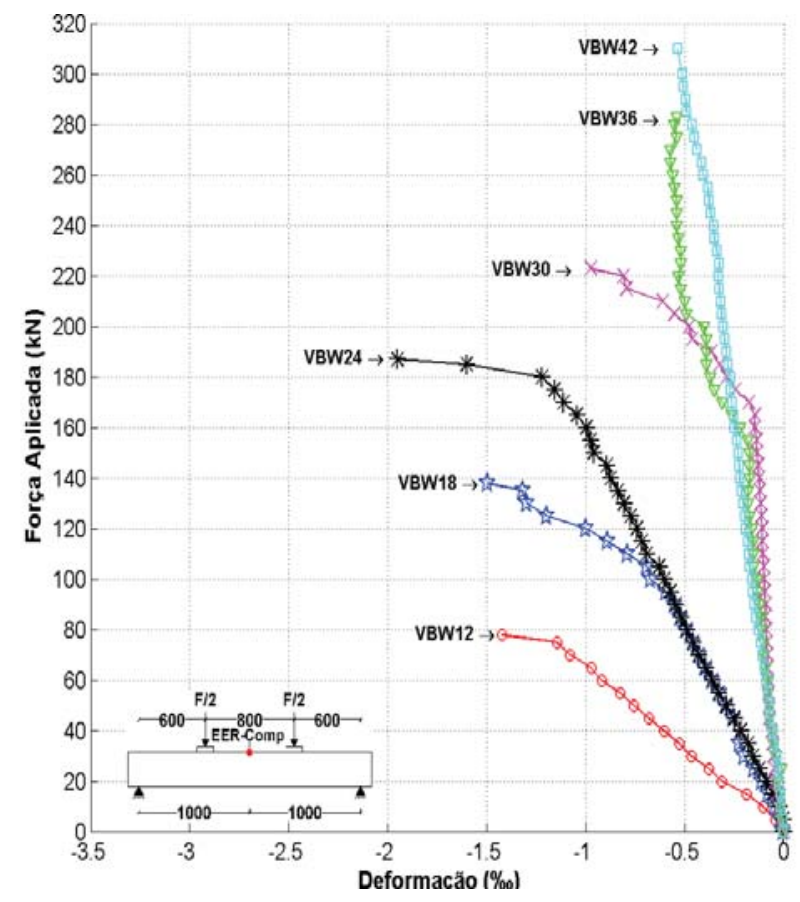

Figura 9: Deformações específicas do concreto

\subsection{Deformação da Armadura de Flexão.}

Os valores de deformação específica da armadura longitudinal de tração na seção do meio do vão são apresentados na Figura 10, onde é possível observar a força aplicada $\left(\mathrm{F}_{\mathrm{ys}}\right)$ responsável pelo escoamento da armadura longitudinal de cada viga, a partir do valor de عys encontrado anteriormente para os corpos-de-prova, estas forças foram apresentadas na Tabela 4 . 


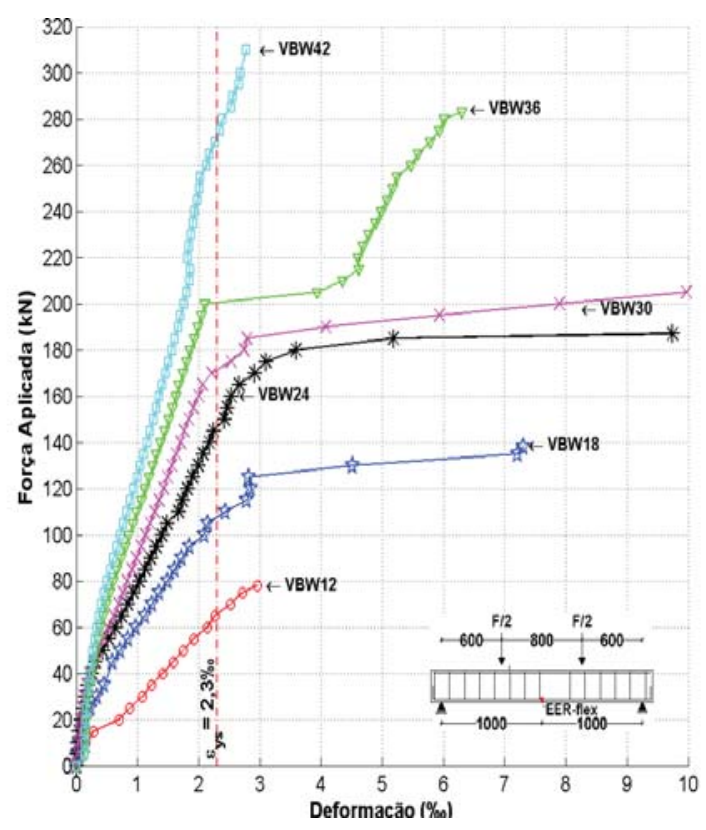

Figura 10: Deformações específicas da armadura de flexão.

\subsection{Deformação Específica da Armadura de Cisalhamento}

Os diagramas força aplicada x deformação específica dos estribos são mostrados nas Figuras 11 e 12, onde é possível observar a força aplicada $\left(\mathrm{F}_{\mathrm{yCis}}\right)$, responsável pelo escoamento da armadura de cisalhamento, a partir do valor de $\varepsilon_{\mathrm{ys}}$ encontrado anteriormente para os corpos-de-prova, estas forças foram apresentadas na Tabela 4. 

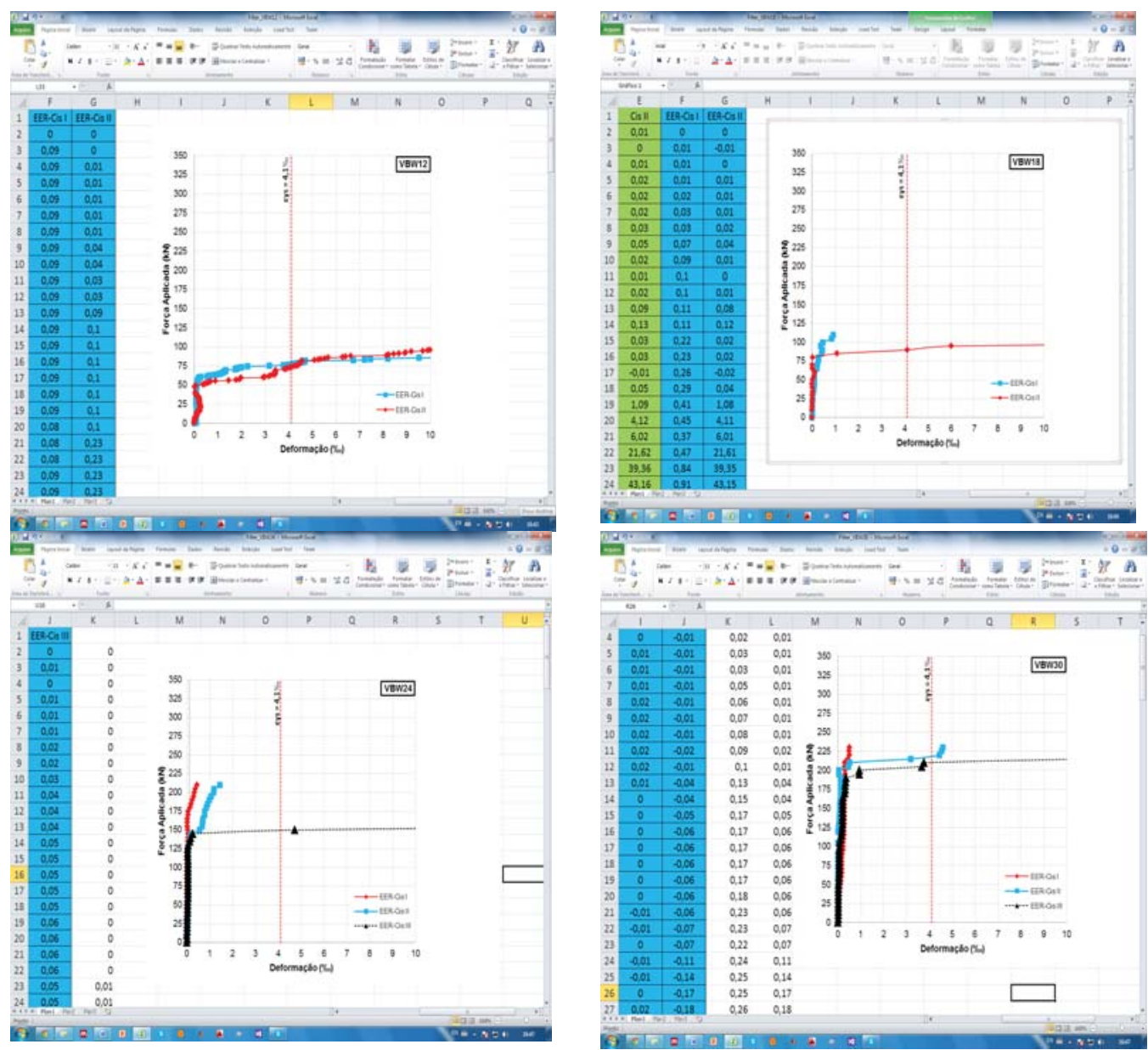

Figura 11: Deformações da armadura de cisalhamento para as vigas VBW12, VBW18, VBW24 e VBW30. 

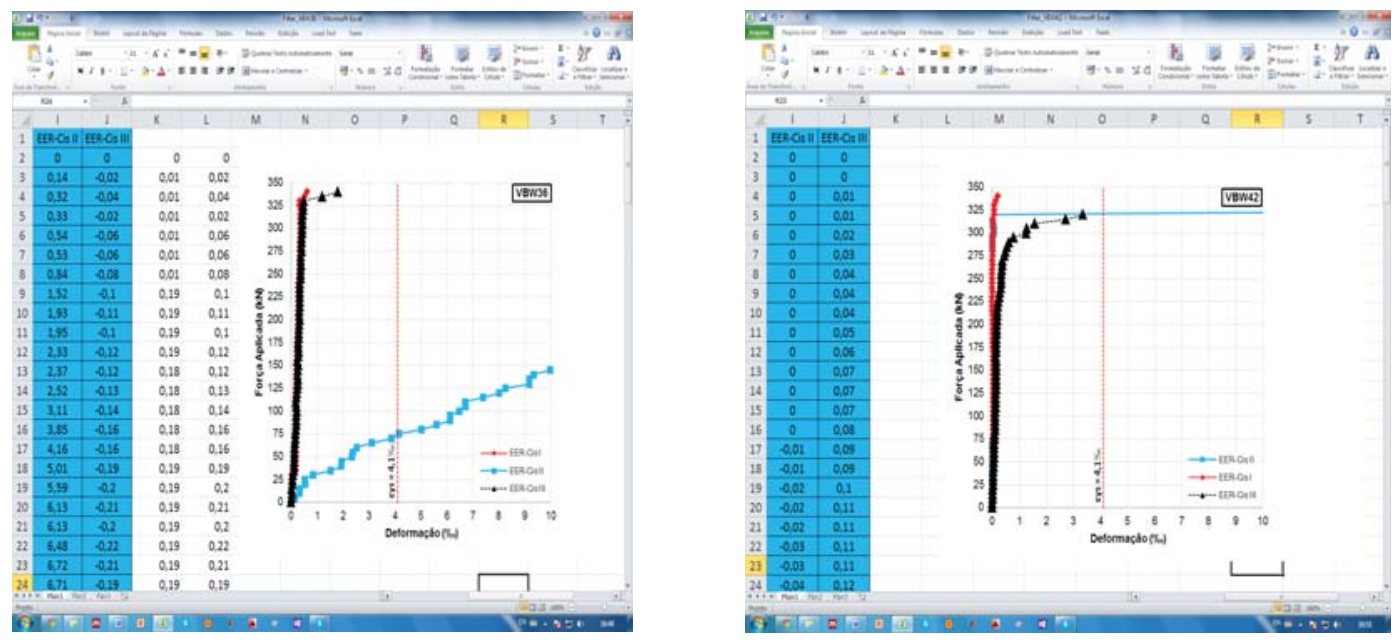

Figura 12: Deformações da armadura de cisalhamento para as vigas VBW36 e VBW42.

\subsection{Forças Últimas e Modos de Ruína}

As forças últimas calculadas de acordo com as normas abordadas neste trabalho e os modos de ruína são apresentados na Tabela 5 , sendo que se optou por calcular tanto o modelo II da NBR 6118 (2007) quanto o Eurocode 2-1(2003) com o valor de 30 para o ângulo de inclinação das bielas comprimidas.

A Figura 13 mostra a comparação entre os valores calculados, segundo as normas, e o valor observado nos ensaios das vigas com bw variando de $120 \mathrm{~mm}$ a $420 \mathrm{~mm}$, com acréscimos de $60 \mathrm{~mm}$. De posse do valor da relação $\left(\mathrm{fu}_{\mathrm{Exp}} / \mathrm{fu}_{\mathrm{Calc}}\right)$, é possível avaliar, segundo a escala de Demérito de Collins (2001), Tabela 6 que, apesar de serem normas com prestígio internacional, ainda divergem significativamente quanto ao valor do cortante máximo resistido por estas vigas.

Tabela 5: Forças (kN) e modos de ruptura.

\begin{tabular}{|c|c|c|c|c|c|c|c|}
\hline Viga & $f u_{\text {Exp }}$ & fys. Exp & $\begin{array}{l}\text { fu. NBR6118 } \\
\text { Modelo I }\end{array}$ & $\begin{array}{c}\text { fu. } \\
\text { MBR6118 } \\
\text { Modelo II (30 }\left(30^{\circ}\right)\end{array}$ & fu. Eurocode $2\left(30^{\circ}\right)$ & fu. ${ }_{A C 1318}$ & Modo de ruína \\
\hline VBW12 & 78,0 & 68 & 45,19 & 64,02 & 30,98 & 41,88 & \multirow{6}{*}{ Cisalhamento } \\
\hline VBW18 & 138,0 & 107 & 67,41 & 96,45 & 45,82 & 62,41 & \\
\hline VBW24 & 188,0 & 145 & 88,39 & 130,28 & 58,51 & 81,55 & \\
\hline VBW30 & 225,8 & 173 & 114,21 & 158,66 & 79,59 & 106,08 & \\
\hline VBW36 & 287,0 & 201 & 137,80 & 189,55 & 96,80 & 128,12 & \\
\hline VBW42 & 310,0 & 270 & 158,16 & 224,08 & 108,42 & 146,58 & \\
\hline
\end{tabular}




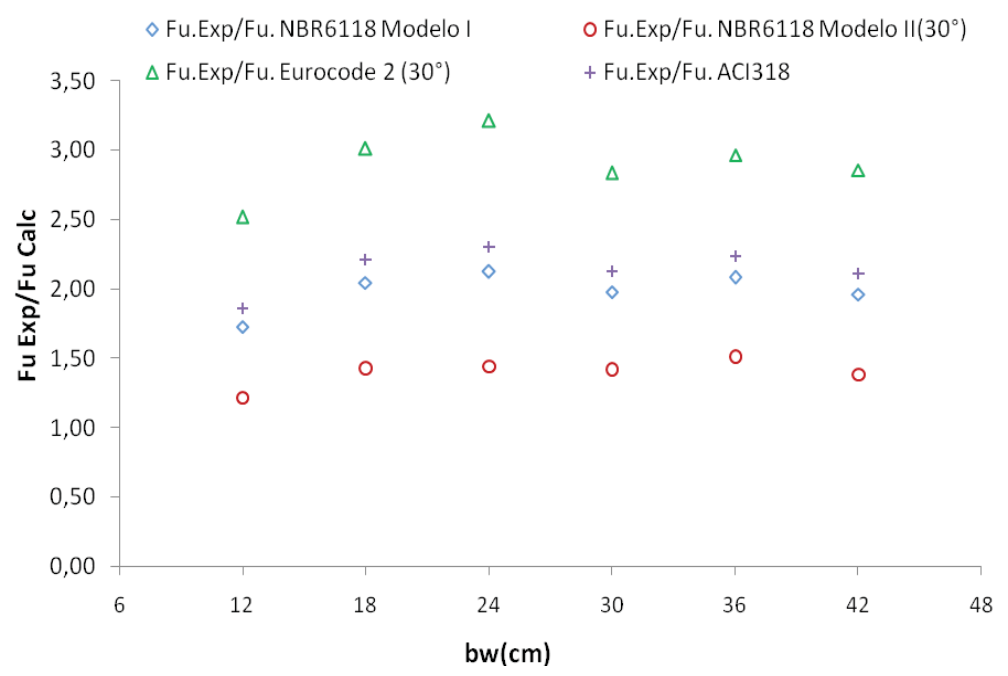

Figura 13: Razão entre a força última experimental e a força estimada.

Tabela 6: Escala de Demérito Collins (2001) apud Ribeiro (2005).

\begin{tabular}{|c|c|c|}
\hline$\tau_{\text {EXP }} / \tau_{\text {CALC }}$ & Escore & Classificação \\
\hline$<\mathbf{0 , 5 0}$ & 10 & Extremamente Perigosa \\
\hline $\mathbf{0 , 5 0} \mid---\mathbf{0 , 6 5}$ & 5 & Perigosa \\
\hline $\mathbf{0 , 6 5}----\mathbf{0 , 8 5}$ & 2 & Baixa Segurança \\
\hline $\mathbf{0 , 8 5}----\mathbf{1 , 3 0}$ & 0 & Segurança Apropriada \\
\hline $\mathbf{1 , 3 0} \mid---\mathbf{2 , 0 0}$ & 1 & Conservativa \\
\hline $\mathbf{2} \mathbf{2 , 0 0}$ & 2 & Extremamente Conservativa \\
\hline
\end{tabular}

\subsection{Fissuração}

Os padrões de fissuração das vigas são mostrados nas Figuras 14 e 15. Para todas as vigas ensaiadas a fissuração iniciou com fissuras de flexão no meio do vão e se propagou em direção aos apoios, sendo perceptível o aumento do número de fissuras com $o$ aumento da base da viga $\left(b_{w}\right)$ antes do seu limite de resistência $\left(f_{\text {uexp }}\right)$. 

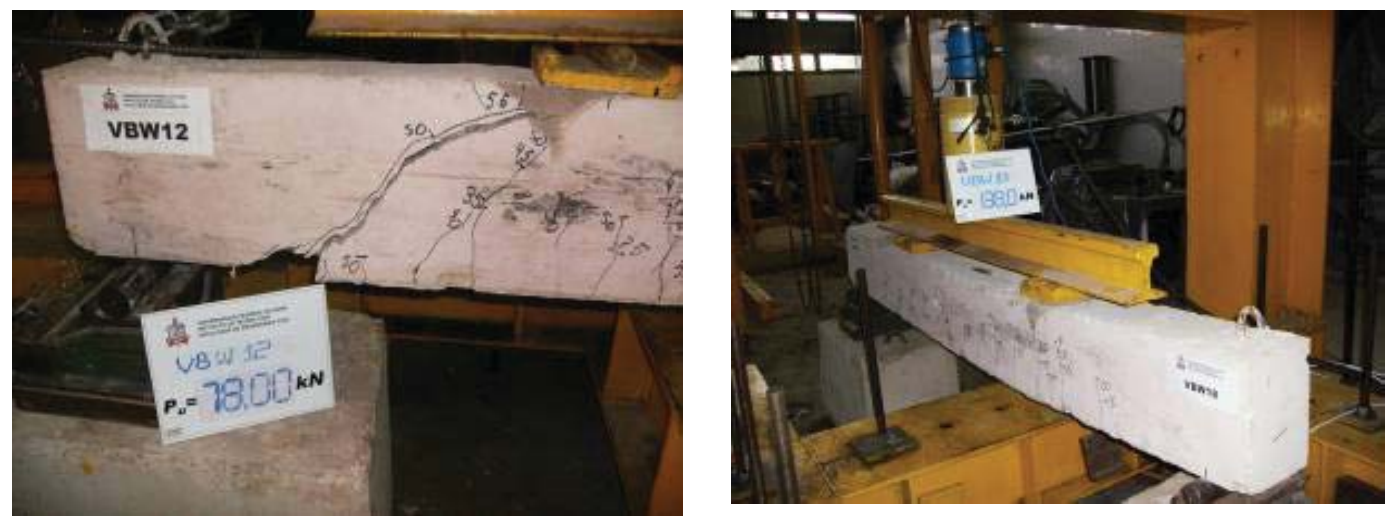

Figura 14: Vigas VBW12 e VBW18 fissuradas.
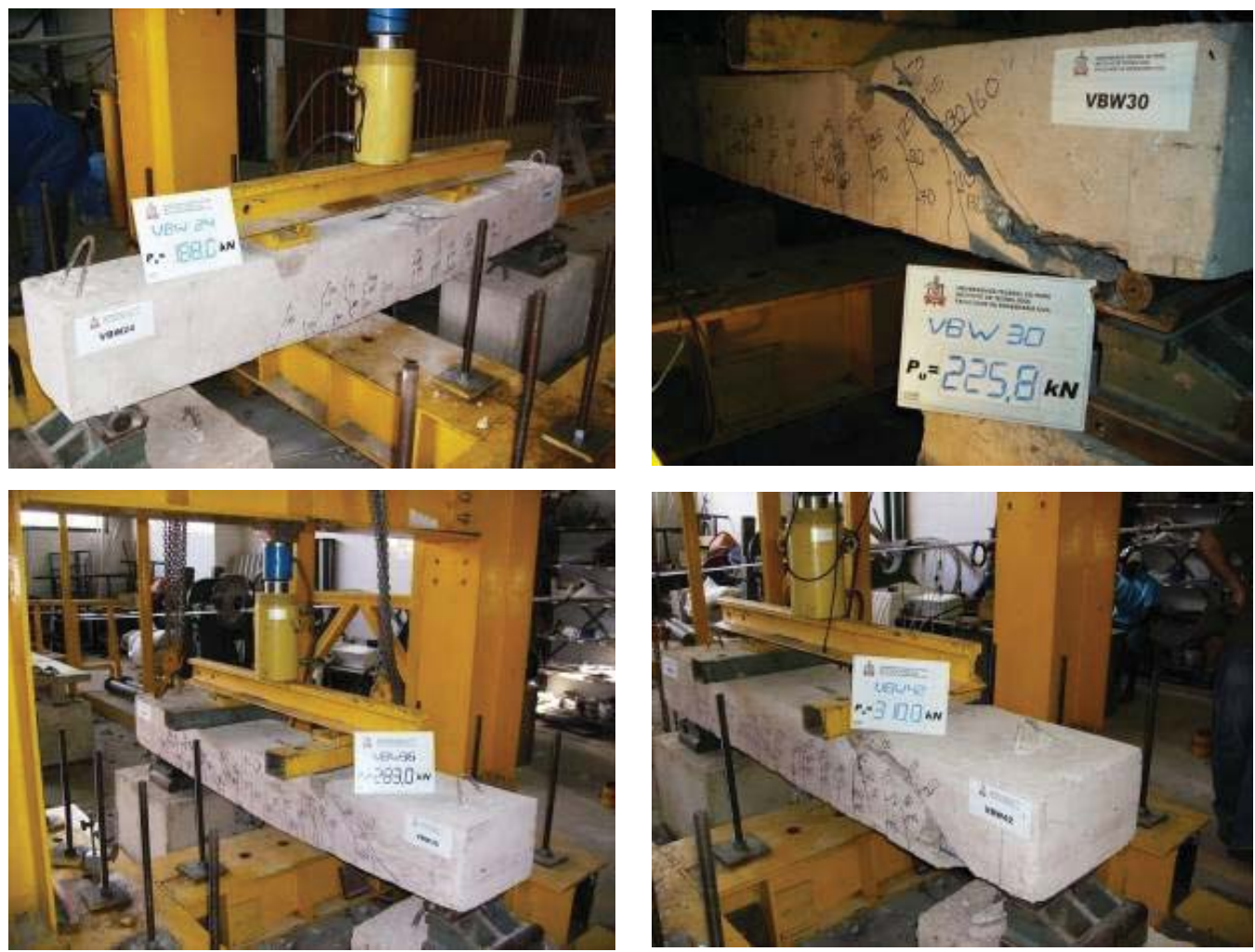

Figura 15: Vigas VBW24, VBW30, VBW36 e VBW42 fissuradas. 


\section{Proposta para Melhorar as Estimativas da NBR 6118 (2007)}

Após analise de diversos casos e confrontado com os resultados experimentais apresentados neste trabalho, foi possível a elaboração de uma nova formulação para o dimensionamento de vigas de concreto armado ao cisalhamento, que faz a utilização da taxa de armadura transversal mínima. A seqüência lógica para a elaboração desta metodologia é apresentada abaixo, Equação (2), Equação (3) e Equação (4). A Figura 16 mostra uma comparação entre as cargas últimas previstas para as vigas ensaiadas e as de diversos autores, encontradas no trabalho de Ribeiro (2005), foram utilizadas vigas possuíam como características obrigatórias, taxa de armadura transversal igual a 1 e relação $b_{w} / d$, entre 0,5 e 1,5 .

$$
\begin{gathered}
\rho_{s w, \min }=\frac{A_{s w}}{b_{w} \cdot s \cdot \cos \theta} \geq \frac{0,06 \cdot f_{c k}^{2 / 3}}{f_{y s w} \cdot \operatorname{sen} \alpha} \\
s=\frac{A_{s w} \cdot f_{y s w} \cdot \operatorname{sen} \alpha}{b_{w} \cdot 0,06 \cdot f_{c k}^{2 / 3} \cdot \cos \theta} \\
\mathrm{V}_{\mathrm{R}}=0,15 \cdot \mathrm{f}_{\mathrm{ck}}{ }^{2 / 3} \cdot \mathrm{b}_{\mathrm{w}} \cdot \mathrm{d}
\end{gathered}
$$

Onde:

Asw = armadura transversal; $\rho$ w,min = é a taxa de armadura transversal minima; $\theta=$ é o ângulo de inclinação das bielas comprimidas;

$\mathrm{s}=$ é o espaçamento dos estribos, em $\mathrm{cm}$;

$\mathrm{b}_{\mathrm{w}}=$ é a menor largura da seção, medida em cm;

$\mathrm{VR}=$ é a força resistente de calculo em $\mathrm{kN}$.

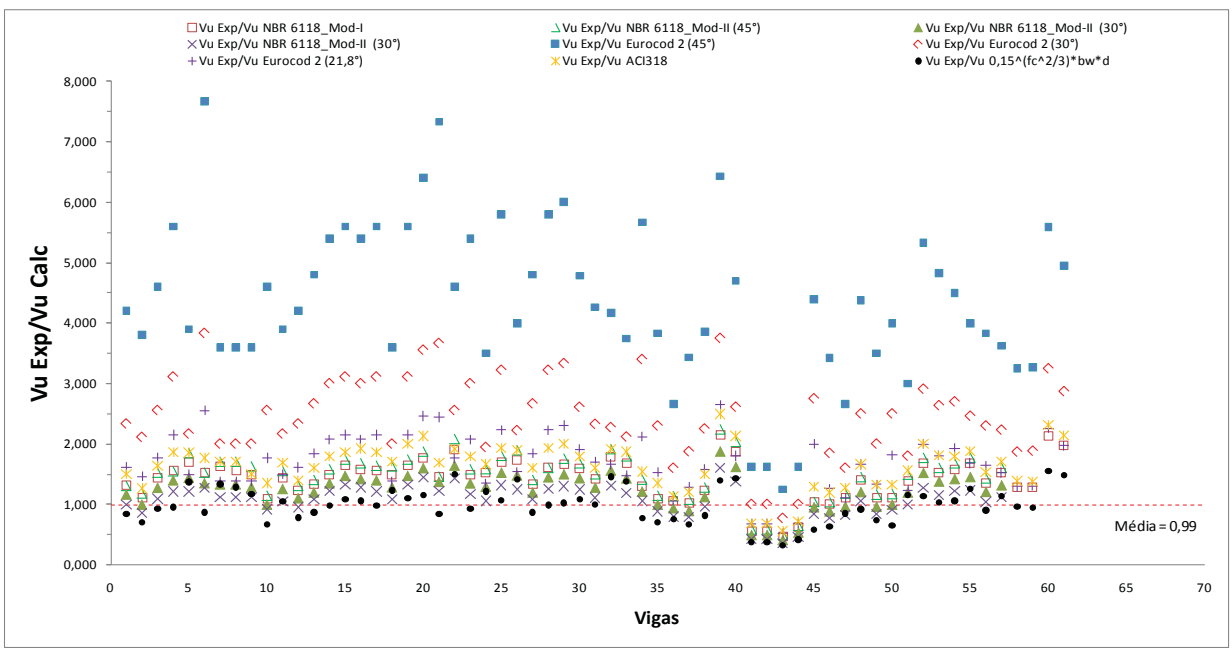

Figura 16: Verificação com as formulações adotadas neste trabalho. 


\section{Conclusões}

As expressões propostas nas normas de cálculo de estruturas de concreto para determinar a taxa mínima de armadura transversal de vigas levam a valores desta taxa significativamente diferentes, tendendo esta diferença a ser maior para concretos de maior resistência. As vigas ensaiadas comportaram-se de maneira compatível com a teoria estudada e foram de fundamental importância para a proposição da nova formulação. Foi verificado também que, por se tratar de formulações empíricas, as normas estudadas neste trabalho divergem significativamente quando comparadas com os resultados experimentais.

\section{Agradecimentos}

Os autores agradecem ao CNPq, CAPES, FAPESPA e ITEGAM pelo apoio financeiro a esta e outras pesquisas desta natureza realizadas na Região Norte do Brasil.

\section{Referências}

Associação Brasileira de Normas Técnicas - ABNT (2007). Concreto - Ensaio de Compressão de Corpos-de-prova Cilíndricos - Método de Ensaio. NBR 5739. Rio de Janeiro.

Associação Brasileira de Normas Técnicas - ABNT (2008). Concreto - Determinação do módulo estático de elasticidade à compressão. NBR 8522. Rio de Janeiro.

Associação Brasileira de Normas Técnicas - ABNT (2007). Projetos de Estruturas de Concreto Procedimentos. NBR 6118. Rio de Janeiro.

Associação Brasileira de Normas Técnicas - ABNT (2002). Materiais metálicos - Ensaio de tração à temperatura ambiente. NBR ISO 6892. Rio de Janeiro.

American Concrete Institute (1995). Building Code Requirements for Structural Concrete. ACI 318. New York.

European Committee for Standardization (2003). Eurocode 2: Design of Concrete Structures Part 1: General Rules and Rules for Building, Brussels. British Standards Institution, London, United Kingdom.

Garcia, S. L. G. (2002). Taxa de armadura transversal mínima em vigas de concreto armado. Dissertação de Mestrado, COPPE/UFRJ, Rio de Janeiro.

Ribeiro, A. B. (2003). Análise crítica sobre o dimensionamento ao cisalhamento em vigas de concreto armado segundo a NBR 6118 (2003). Dissertação de Mestrado, UFMG, Belo Horizonte. 


\title{
Influence of transverse reinforcement recommended by NBR 6118 (2007), ACI 318 (2005) and Eurocode 2-1 (2003) codes on the behaviour of reinforced concrete beams
}

\begin{abstract}
Aiming to evaluate the minimum transversal reinforcement's influence on the behaviour of 6 reinforced concrete beams of 2,200 mm length and rectangular cross section of $250 \mathrm{~mm}$ height and width dimensions varying from 120 to $420 \mathrm{~mm}$ were cast and tested under shear at the Civil Engineering Laboratory of the Federal University of Para. Flexural reinforcement and the $2,000 \mathrm{~mm}$ span were kept constant. Results for failure loads and failure modes, steel and concrete strains and vertical displacements are presented and discussed. The experimental results showed that the NBR 6118 (2007) Code diverges significantly when the width varies.
\end{abstract}

Keywords: Reinforced concrete. Beam. Shear.

\section{Introduction}

In the design of structural elements whose actions are mainly shear forces is essential a thorough analysis about their ductility, so that in case of any imminent accident the structure could "advise" whether the ultimate strength is close or not, either to give time for evacuation, if necessary, or to adopt reasonable measures to ensure that the structure will be retrofitted.

According to Garcia (2002), the minimum transverse reinforcement of beams should be provided to avoid brittle failure when the diagonal cracking occurs, in case of the existence of unforeseen overloads, limiting the concrete strut slope and the opening of inclined cracks and also preventing buckling of the compressed longitudinal reinforcement.

The codes intended for the calculation of reinforced concrete structures set a minimum transverse reinforcement, but not all of them clarify their goals or include any notes in which these definitions for reinforcement are relied on. The ACI-318 (2005) code states that the minimum transverse reinforcement restricts the development of the inclined cracking, increases ductility of the beam and provides remarks of a possible failure, whose reasons are of particular importance for pieces subjected to unforeseen overloads.

Discussions focused on the review and adjustment of the minimum rate of transverse reinforcement motivated this work that dealt with an experimental analysis of beams by using minimum rate of reinforcement and different $b_{w}$. In addition, a new formulation for the calculation of the ultimate shear strength of reinforced concrete beams with minimum shear reinforcement is presented. 


\section{Code prescriptions}

Table 1 shows the formulations adopted by each one of the analysed codes for the shear strength design of reinforced concrete beams with stirrups perpendicular to the member's longitudinal axis. Both NBR 6118 (2007) and Eurocode 2-1 (2003) use as a principle the Mörsch generalized truss model. These codes allow the angle $\alpha$ between shear reinforcement and the main tension chord (transverse reinforcement) vary from $45^{\circ}$ to $90^{\circ}$ and prescribe $45^{\circ}$ as the maximum value for the angle $\theta$ for the concrete compression strut. Eurocode 2-1 (2003) allows the use of $\theta$ with a minimum value of $21.8^{\circ}$ while the NBR 6118 (2007) limits this minimum value at $30^{\circ}$. The value of the angle $\theta$ is arbitrated by the designer. When checking the failure by diagonal tension, it is observed that the Eurocode 2-1 (2003) does not take into account the contribution of the complementary mechanisms of the truss, i.e., the transverse reinforcement fully absorbs all the shear resistance. On the other hand, ACI 318 (2005) is based on the classic truss analogy whose the inclination angle $\theta$ of the compression strut is fixed at $45^{\circ}$.

\section{Methodology}

To evaluate the influence of the minimum rate of transverse reinforcement $\left(\mathrm{A}_{\mathrm{sw}}\right)$ for different values of $b_{w}$ in the ultimate behaviour of reinforced concrete beams with rectangular section, 6 reinforced concrete beams with dimensions of 2,200 $\mathrm{mm}$ in length $(\mathrm{L}), 250 \mathrm{~mm}$ for the height $(\mathrm{h})$ and base $\left(\mathrm{b}_{\mathrm{w}}\right)$ ranging $120-420 \mathrm{~mm}$ with an increment value equal to $60 \mathrm{~mm}$ were analysed experimentally These and other specifications of the beams are shown in Figures 1 and 2 and other relevant specifications of the test pieces are shown in Table 2.

\section{Proposal to Improve Estimates of NBR 6118 (2007)}

After analysing several cases and confronted them with experimental results presented in this work, it was possible to draw up a new formulation for the design of shear in reinforced concrete beams, by using the concept of the minimum rate of transverse reinforcement. The logical sequence for the development of this methodology is presented below, Equation (2), Equation (3) and Equation (4). Figure 16 shows a comparison between the estimated ultimate loads for the tested beams and various authors, found in the work of Ribeiro (2005), in which beams used as mandatory characteristics the transverse reinforcement ratio equal to 1 and the $b_{w} / d$ ratio between 0.5 and 1.5. 


\section{Conclusions}

The proposed expressions presented in the codes for the calculation of concrete structures used to determine the rate of minimum shear reinforcement for beams lead to significantly different values, tending to a greater difference the higher is the concrete strength. The tested beams behaved consistently with the theoretical behaviour and they were of fundamental importance to the proposition of a new formulation. It was also found that, since it is dealt with empirical formulations in this work, the codes studied differ significantly when compared with the experimental results. 